\title{
Modeling of post-traumatic epilepsy and experimental research aimed at its prevention
}

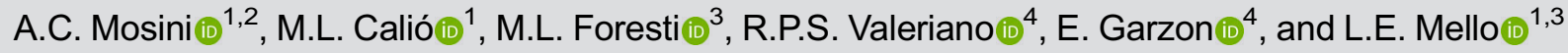 \\ ${ }^{1}$ Departamento de Fisiologia, Universidade Federal de São Paulo, São Paulo, SP, Brasil \\ ${ }^{2}$ Associação Brasileira de Epilepsia, São Paulo, SP, Brasil \\ ${ }^{3}$ Instituto D'Or de Pesquisa e Ensino, Rio de Janeiro, RJ, Brasil \\ ${ }^{4}$ Divisão de Clínica Neurológica, Faculdade de Medicina, Universidade de São Paulo, São Paulo, SP, Brasil
}

\begin{abstract}
Research on the prevention of post-traumatic epilepsy (PTE) has seen remarkable advances regarding its physiopathology in recent years. From the search for biomarkers that might be used to indicate individual susceptibility to the development of new animal models and the investigation of new drugs, a great deal of knowledge has been amassed. Various groups have concentrated efforts in generating new animal models of traumatic brain injury (TBI) in an attempt to provide the means to further produce knowledge on the subject. Here we forward the hypothesis that restricting the search of biomarkers and of new drugs to prevent PTE by using only a limited set of TBI models might hamper the understanding of this relevant and yet not preventable medical condition.
\end{abstract}

Key words: Epileptogenesis; Animal models of epilepsy; Traumatic brain injury; Status epilepticus; Kindling; Seizure

\section{Introduction}

Traumatic brain injury (TBI) is a global significant cause of death and lifelong disability resulting in cognitive, physical, behavioral, and subjective sequela. It has been estimated that TBI affects over 10 million people annually worldwide, leading to either high mortality or hospitalization rates (1). Age-standardized TBI prevalence has grown $8.4 \%$ from 1990 to 2016 (2), and it is still considered a silent epidemic (3). Among younger adults, especially men, TBI is a major source of years lived with disability. In addition, reflecting the ageing global population, even more so in high-income countries, in recent decades there has also been an increase in the number of TBI occurrence in elderly people, mainly related to falls (2).

TBI is defined as an alteration in brain function, or other evidence of brain pathology, caused by an external mechanical force, such as an impact, rapid acceleration or deceleration, blast waves, crush, or penetration by an object, resulting in focal or diffuse types of injury (4). As presented, TBI is a heterogeneous condition, which embraces different causes, severity levels, and consequently a wide range of prognosis. Patients with moderate to severe TBI have a high overall mortality, and more than $40 \%$ of survivors experience long-term disabilities (5). Even mild TBI has been associated with several long-term adverse outcomes. Disabilities can manifest as physical and cognitive deficits (for example, transient confusion, disorientation, and impaired attention), psychological health issues (such as depression), and impairments in self-regulatory behaviors (such as increased impulsivity, poor decisionmaking, and aggressive behavior) (3). TBI is also the main cause of acquired epilepsy (6).

Epilepsy is defined as a disorder of the brain characterized by an enduring predisposition to generate epileptic seizures, and by the neurobiological, cognitive, psychological, and social consequences of this condition (7). Posttraumatic epilepsy (PTE) accounts for $5 \%$ of all epilepsy etiologies, making TBI one of the most important causes of secondary epilepsy, overcoming other causes such as infections and central nervous system tumors (8).

In spite of the high PTE incidence after TBI, the latency from injury to development of epilepsy is variable and can range from weeks to years (9). Seizures occurring in the first 1-2 weeks after trauma may be due to the acute effects of the trauma, such as hemorrhage and brain edema, and do not necessarily characterize epilepsy. Even so, the presence of acute seizures occurring within weeks after TBI is often thought to be associated with the progression to PTE further along in life. The recurrence of epileptic seizures within the first 2 years in TBI patients with a single acute seizure after trauma might be as high as $86 \%$ 
(10). The causative links between TBI and epilepsy, as well as other types of epilepsy in general, are still not completely understood, and PTE is not yet preventable.

Epileptogenesis is a dynamic process occurring between the initial brain insult and the onset of epilepsy, characterized by the development of the first spontaneous seizure. The epileptogenic process can be divided into three different stages: 1) Initial insult: primary injury by different insults, including the already mentioned TBI, and also status epilepticus (SE), febrile seizures, stroke, craniotomy, infections, tumors, and neurodegenerative diseases, among others; 2) Latent period: the primary injury triggers a cascade of molecular, cellular, and structural changes that include increased neuronal excitability and inflammatory processes, which contribute to additional neurodegeneration, and reorganization of molecular and cellular pathways, including gliosis, plasticity, neurogenesis, and mossy fiber sprouting $(11,12)$. All these processes are supposed to be related, with greater or lower importance, to the development of the epileptic focus; and 3) Chronic epilepsy phase: clinical manifestation of spontaneous recurrent seizures.

Therefore, different insult types can be used to mimic specific characteristics of the primary lesion, and the subsequent epileptogenic mechanisms can be further studied during the latent period in different models of PTE.

The goal of this review is to describe the main animal models currently used for the study of PTE and to critically compare those models with regard to the development of the most common features present in this pathology. We propose that the onset of spontaneous and recurrent seizures, regardless of the initial stimulus, should be the most relevant feature of animal models used for the study of PTE. We suggest that the epileptogenesis associated with different etiologies are often very similar and that the investigation of potential means of disease modification or prophylaxis, in the case of PTE, can rely not only on TBI models, but also on a broader set of epilepsy models.

\section{Animal models of post-traumatic epilepsy}

It is a great achievement for science to reproduce human diseases in animal models. A good animal model is homologous, able to mimic the human disorder and the physiopathological mechanisms in every respect. Alternatively, the animal model can be isomorphic, when it duplicates the disorder but not the underlying etiology, or predictive, in the case of models that cannot reproduce the human disorder but allow predictions about it or its response to treatment (13). As posed by the great mathematician Norbert Wiener "the best material model of a cat is another, or preferably the same, cat" (https://en. wikiquote.org/wiki/Norbert_Wiener). This review's whole argument is that, depending on the focus, the best model should mimic mechanisms and not form. To that end, here we evaluate both models of epileptogenesis, consisting of either repetitive stimulation of specific brain structures (kindling models) or the induction of intense prolonged acute seizures (SE models), as well as models developed for the study of PTE, using mechanical injuries (TBI models) or iron deposition (mimicking intraparenchymal bleeding). Other animal models for studying the epileptogenesis process, such as febrile seizures, models with transgenic animals, and stroke will not be explored in the current review. Indeed, this would not only substantially alter the scope of the current revision but would also lead to loss of the main argument we want to raise. Most groups using febrile seizures to study epilepsy are interested in developmental aspects of this condition and preferentially deploy juvenile animals. Similar limitations arise from the use of transgenic animals, which are usually structured as a means to investigate very specific contributions of a given protein or systems.

\section{TBI models}

Animal models more directly related to TBI mechanisms were developed to investigate how the natural progression of trauma contributes to epileptogenesis. In a pioneer work, Willmore et al. were able to induce spontaneous seizures in animals over a period of a few weeks, by using a single iron injection to different brain regions (14). This injury model importantly relates to intracerebral hemorrhage toxicity caused by iron-rich hemoglobin breakdown products, similarly to what happens after a brain trauma or hemorrhagic stroke. It is known that $\mathrm{FeCl}_{2}$ does induce free radical formation, lipid peroxidation, and edema that can be attenuated by antiperoxidants. $\mathrm{FeCl}_{2}$ also causes alterations in glutamate transport (15). Recurrent seizures and epileptic discharge similar to human PTE have been seen after the generation of free radicals with the intracortical administration of ferric chloride into the sensorimotor cortex (16).

Thereafter, additional models mimicking different types of mechanical trauma have gained increased attention among experts investigating epileptogenesis associated with TBI. Among these, the fluid percussion injury (FPI), controlled cortical impact $(\mathrm{CCl})$, weight-drop impact acceleration injury, and blast injury can be highlighted (4). Only a few models consider the importance of penetrating injury to epileptogenesis, such as the penetrating ballistic-like brain injury (17).

The lateral FPI model is the most utilized mechanical model to study PTE. By using a closed hydraulic system, a precise pressure pulse is delivered distinctly in the animal dura mater, according to the intended injury severity (18). In this model, a single event of lateral FPI is sufficient to induce chronic spontaneous recurrent partial seizures that worsen over time (19). According to D'Ambrosio and Perucca, lateral FPI can develop two epileptic foci depending on its location, one in the neocortex adjacent to the impact site that defines the early epileptic manifestations 
after injury, and one in the hippocampal region, which becomes increasingly dominant over time.

It is an easy to induce and highly reproducible model, but it has limitations such as high mortality in the severe injury conditions necessary to generate spontaneous seizures, increasing the total number of animals required for the PTE study. This model also promotes a low seizure frequency, and thus requires prolonged time of electroencephalogram (EEG) monitoring of spontaneous seizure events (20).

Another model used to directly mimic $\mathrm{TBI}$ is the $\mathrm{CCl}$ injury that was described by Lighthall (21) using ferrets as the test animal. During the 1990s, the model was adapted to be used in other animals like rats, mice, and primates. This model consists of a brain injury produced by a pneumatic or electromagnetic impact that compresses the exposed brain and results in brain injury with varying severity (18). Similar to FPI this model was created as a model of TBI and adapted for studying PTE.

As in the case of FPI, scarce late spontaneous seizures or epilepsy have been reported in rat $\mathrm{CCl}$ models $(18,22)$. Despite the low frequency of spontaneous seizures, the model also leads to an increase in seizure susceptibility when challenged with other agents (23).

The $\mathrm{CCl}$ model provides consistency, reproducibility, with a low mortality rate compared to lateral FPI, being beneficial in reducing sample attrition and keeping study cost down. In contrast, this model includes mechanical variation, wear on the device, limited diffuse effects, and most importantly, it results in a low frequency of spontaneous chronic seizures, making it less useful for the study of epileptogenic mechanisms.

Impact-acceleration, also known as the weight drop model, is also a model of diffuse TBI. The animal is positioned on a foam block to provide a consistent position of the animal's body and a pre-selected weight is dropped from a pre-selected height according to the desired injury severity. The weight strike promotes not just a focal injury, but also promotes an acceleration-deceleration of the animal's head into the foam block (4).

The impact-acceleration model produces mild-to-moderate convulsions during its acute phase and may also cause late convulsions lasting up to 15 weeks post-injury. Although the model demonstrated an increase in susceptibility to pentylenetetrazol (PTZ)-evoked seizures, it fails to adequately recapitulate PTE because, without using a high intensity impact that frequently promotes great mortality, it does not promote spontaneous seizures (18). On the other hand, this model presents the advantages of simplicity of the method, successfully inducing a reproducible lesion and consistently increasing susceptibility to PTZ-induced seizures.

Another important model of TBI is the blast model that mimics a real blast-induced mild TBI, such as seen in military conflicts, and can be a potential risk factor for behavioral disorders, cognitive alterations, and for the development of neurodegenerative disorders. According to Song et al. (24), animals are allocated in open or closed shock tubes, or in an open field, $30 \mathrm{~m}$ away from a TNT explosive. Neocortical, hippocampal, and cerebellar tissue injuries have been reported in different degrees in the blast model together with vascular injuries, hemorrhage, and diffuse axonal injury (24). In the blast model, the occurrence of EEG epileptiform or PTE have not been generally assessed. Only a few studies show the occurrence of acute seizures following severe blast and there are no published studies investigating the occurrence of chronic (spontaneous) seizures or epileptogenic alterations in this model (25). The main advantage of the blast model is that it closely replicates the scenario of a realworld blast. On the other hand, this model is not ideal to investigate PTE, as it is expensive, it is difficult to find a safe place to store explosives, and the technical time demand and the environmental conditions such as temperature and wind may influence the animal responses and the blast setting (24).

Similarly, in the penetrating ballistic-like injury model, electrographic waveforms of ictal and inter-ictal events are reported, but occur later after injury and less frequently compared to those occurring in other injury models (26). In the repetitive weight-drop model and in the repetitive blast model, only a subset of mice (44 and $46 \%$, respectively) develop spontaneous recurrent seizures (27).

As aforementioned, spontaneous epileptic activity, when present after mechanical injury, tend to show milder characteristics in terms of the epileptic events in TBI models (Figure 1). For example, the epileptogenesis phase in the FPI injury is longer, the resulting frequency of spontaneous seizures is substantially lower and occurs in fewer animals, and most of the seizures are secondarily generalized rather than partial, compared to epilepsy models that induce SE as the precipitating injury (28).

\section{Kindling model}

One of the first and still widely used animal models to study epileptogenesis is the kindling model, which is characterized by the progressive development of focal seizures to secondarily generalized tonic seizures, after repeated application of an initially subconvulsive stimulus (29), which can be either electrical or chemical.

Electrical kindling applies subthreshold repetitive electrical stimuli in specific brain regions such as the dorsal hippocampus, olfactory bulb, amygdala, perirhinal cortex, and perforant path (30). Normally, these subthreshold stimuli, when administered occasionally, do not result in behavioral convulsive episodes, but, when administered repetitively, can promote behavioral convulsions. Rather than using electrical stimuli, chemical kindling involves repetitive administration of chemical convulsants. Convulsant substances such as cocaine, N-methyl-D-aspartate, and GABAa receptor antagonists, such as PTZ, can be used to induce chemical kindling. PTZ, one of the most 
widely used agents for the induction of chemical kindling, produces severe self-limited convulsions in animals.

In the kindling model, the seizure focus is explicit, seizures evolve gradually, and the development of chronic epileptogenesis can be investigated while not resulting in severe morphological damage (31). The progressive changes have a permanent feature, and although laborious, repeated stimulation for extended time can result in spontaneous seizures $(32,33)$.

Unfortunately, this method demands resources and time, comprising long periods of handling and stimulation procedures (34). Still, many parameters of the kindling protocol have been modified to increase kindling representativeness as an epileptogenic model and to increase efficiency to assess antiepileptic drugs (AEDs). Such changes include but are not limited to: i) decreasing the interstimulus interval to shorten seizure development from weeks to hours (35); ii) selective breeding for segregating genetic predisposition to differential kindling rates (36); and iii) more recently, the application of the novel optogenetic approach to stimulate animals (37).

\section{SE models}

The induction of SE, which is characterized by acute self-sustained seizures, is another classical experimental approach for the study of epileptogenesis. As in the kindling model, several stimuli can be used to trigger SE, including deep electrical stimulation to specific brain areas and systemic or topical injection of chemical agents, most notably kainic acid (KA) and pilocarpine (38-40). The early phase after the initial precipitating injury is associated with intense neuroplastic changes, neurodegeneration, and neuroinflammation. After a period past the SE event, spontaneous recurrent seizures easily develop together with behavior impairments associated with chronic inflammation, altered neurogenesis, abnormal synaptic reorganization, and multiple molecular changes.

The pilocarpine model consists in the systemic or topical (intrahippocampal) administration of this cholinergic agent, a potent muscarinic agonist. It promotes behavioral and electrographic alterations resulting in three different periods, which resemble the stages of the epileptogenesis process already described: i) Acute period characterized by SE originating in the limbic system and lasting up to $24 \mathrm{~h}$, or until interruption with some specific drug; ii) Latent period, when the progressive normalization of behavior and EEG aspects and epileptogenic changes take place (this period has been reported to vary from 4-44 days); and iii) Chronic period, with the emergence of spontaneous recurrent seizures. The spontaneous seizures observed during the chronic period are similar to the complex partial seizures seen in patients. Seizure frequency in the chronic period is variable, some animals present a low seizure frequency over several weeks or months, while others may have daily seizures, and others may present seizures in short periods of time (41). Yet, all animals undergoing pilocarpine-induced SE develop spontaneous seizures and this is by far the animal model of epilepsy with the highest frequency of those seizures (Mello LE, unpublished results).

Another important SE model is the kainic acid model, which results from the intraperitoneal or intrahippocampal administration of $\mathrm{KA}$, an agonist of the ionotropic glutamate receptor and a cyclic analog of L-glutamate. As it occurs after pilocarpine administration, the epileptiform discharges after KA administration originate in limbic structures and then propagate to other brain areas (38), mimicking human SE and promoting EEG changes similar to those seen in patients. As a disadvantage, KA and pilocarpine models result in high animal mortality and variable frequency and severity of spontaneous seizures among individual animals (34).

The maximal electroshock seizure, $6-\mathrm{Hz}$ corneal psychomotor seizure, and pentylenetetrazol injection are other established models to induce acute seizures, but differently from what occurs in SE models, those seizures are self-limited and mostly used to test anti-seizure drugs, instead of PTE prevention.

\section{Models and physiopathological mechanisms}

\section{Neurodegeneration}

Although the mechanisms underlying the development of spontaneous recurrent seizures are not clearly understood, it is known that the degenerative process is one of the characteristics of brain pathologies related to acquired epilepsy (12).

The existence of relatively high numbers of animal models in which the developmental process and the morphological and molecular features of epilepsy in humans are reproduced has obvious advantages for research. Ideally, a model will efficiently reproduce the variety of tissue loss and damage detected in TBI. In human patients with severe TBI, besides local neuronal loss, subcortical neurodegeneration in the thalamus and hippocampus are also observed and may contribute to epileptogenesis (28).

Comparing two SE models, Covolan and Mello (42) observed prominent thalamic degeneration in the pilocarpine as well as in the KA model, although in the pilocarpine model, neuronal injury was more remarkable than that in the KA model in numerous areas in cortex, hippocampus, endopiriform nucleus, amygdaloid complex, and hypothalamus. On the other hand, there is no neuronal damage associated with the electrical kindling model (31). Furthermore, in the model of cortical injection of ferrous chloride, neurodegeneration is limited to the cortex (14). Moreover, the $\mathrm{CCl}$ model of TBI can cause different levels of injury severity and several degrees of 
cortical, subcortical, and hippocampal tissue damage that could lead to substantial neurodegeneration and tissue loss in the ipsilateral neocortex and adjacent hippocampus. The acute injury results in increased neuron loss, considerable lesion volumes, and more severe functional deficits (43) even for a long period (44).

As reviewed here, neurodegeneration is an important feature of almost all animal models considered in this section (Figure 1). There is no specific lesion profile or characteristic that is unique to any of the TBI models. More importantly, there are no neurodegenerative hallmarks that are only present in human PTE, even more so as the lesion event may affect a number of different cortical and subcortical regions.

\section{Neuroplasticity}

As a consequence of brain injury, several neuroplastic changes might ensue. Neural plasticity involves both the proliferation and differentiation of newly-formed cells, in a process called neurogenesis, as well as alterations in the structure and function of neurons at different levels, from their morphology, subunit receptor composition, or neurotransmitter expression to synaptic connections. In this sense, neurogenesis (45) and axonal sprouting (46) have been extensively seen in chemically-induced SE models. The efforts to reestablish the neural connections could be beneficial, but could actually be adverse (or even an epiphenomenon) to the occurrence of spontaneous recurrent seizures in these animal models (46).

Neurogenesis. As previously mentioned, the latent period is a phase that occurs after the initial precipitating injury, lasting from days to weeks, in which the epileptogenic process occurs, leading to the development of later spontaneous seizures. Curiously, it was noticed that during the latent period a dramatic increase in cell proliferation in the subgranular zone of the dentate gyrus of the hippocampus and in subventricular zone of lateral ventricles occurs in several animal models of PTE (47). Jessberger and Parent (48) showed that epileptic activity induced by KA led to changes in the neuronal polarity, migration, and integration pattern of newly born granule cells, resulting in their ectopic location in the hilus. Zellinger

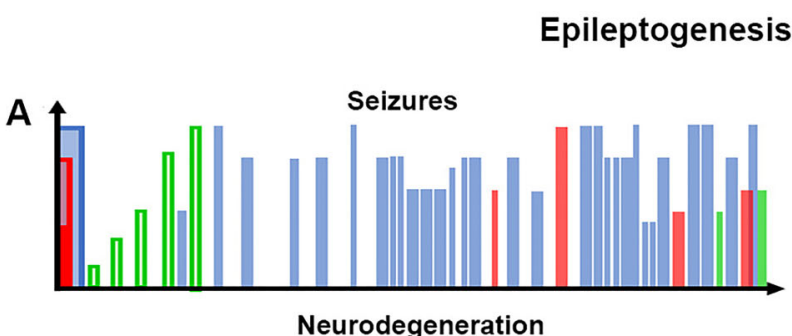

\section{PTE models}

SE

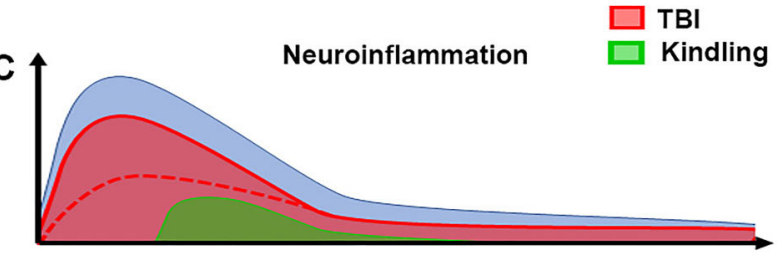

Neuroplasticity

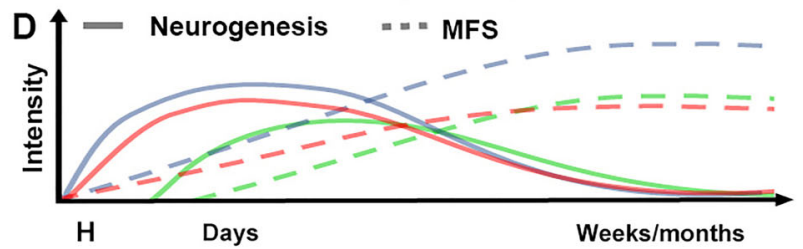

Figure 1. Schematic representation of epileptogenic features in main animal models of post-traumatic epilepsy (PTE). A, In classic models of status epilepticus (SE) (e.g., pilocarpine and kainic acid), after the initial sustained acute seizure, the development of spontaneous recurrent seizures occurs after a brief latent period in most animals. In contrast, the slow progression of evoked seizures in kindling models implies the need of massive repetition of the stimuli protocol in order for spontaneous seizures to develop, making this an extremely laborious protocol for that intention. Meanwhile, different parameters can be adjusted according to the desired lesion severity, and consequent occurrence or not of acute seizures, in different traumatic brain injury (TBI) models (e.g., lateral fluid percussion, controlled cortical impact, impact-acceleration, or weight drop). However, in TBI models, usually only a few animals, most of them requiring severe initial lesions, develop spontaneous recurrent seizures following a longer latent period. B, The lesion event may differently affect neurodegeneration occurrence. In general, chemically-induced SE causes severe and widespread neurodegeneration, while minor neuronal damage is detected in the kindling model. Importantly, by adjusting the mechanical force parameters, the neuronal damage can vary from cortical to subcortical regions, and from minor to severe neurodegeneration in TBI models. C, The neuroinflammatory response is rapidly initiated following the initial precipitating injury, characterized by the release of inflammatory cytokines, chemokines, and complement proteins. Astrocytes and microglial cells also became activated, proliferate, and, together with peripheral immune cells, are recruited to the lesion site. This response decreases over days, but residual neuroinflammation may chronically persist, supporting a pro-epileptogenic role. In general, the intensity of the neuroinflammation response can be considered quite similar between SE and TBI models, varying with the impact intensity in the latter. D, There is a transitory increase in newborn neurons in the subgranular zone of the dentate gyrus of the hippocampus and in the subventricular zone of lateral ventricles, in several animal models of PTE. After this initial increase, the number of newly generated cells returns to basal levels with the occurrence of spontaneous seizures. On the other hand, mossy fiber sprouting (MFS) increases after the initial insult and presents a permanent pattern together with spontaneous seizure occurrence. 
et al. (49) observed that prolonged seizure activity induced by electrical stimulation resulted in a significant rise in the number of neuronal progenitor cells. Moreover, a significant increase in neurogenesis was observed in the granule cell layer of the dentate gyrus following pilocarpine-induced SE (45) and kindling stimulations (47).

Indeed, seizure activity is a neurogenic stimulus. Increased hippocampal neurogenesis is a common hallmark of most SE models, as well as TBI models (50) that develop epilepsy (Figure 1). Approximately 4-6 weeks after the initial insult, cell proliferation returns to normal levels $(47,51)$.

Neuberger et al. (52) showed that the early increase in neurogenesis after FPI is transitory and is followed by a persistent and dramatic decrease in the number of newly generated cells. They suggest that early post-traumatic increases in neurogenesis negatively affect long-term events by diminishing the neurogenic potential of neural stem cells, which might in turn contribute to epileptogenesis. In addition, Hattiangady et al. (53) demonstrated that the intensity of neurogenesis declines in the chronic phase with the occurrence of spontaneous seizures.

It is believed that excessive neurogenesis may contribute to an aggravation of pathology, causing aberrant connectivity and enhanced excitability (54) and although seizures enhance neurogenesis, the survival of the newborn granular cells may decrease with increased seizure severity due to microglial activation (55).

While much work has been conducted regarding the neurogenic process in response to injury in animal models, only a few studies demonstrate these results in humans after TBI. In this sense, Zheng et al. (56) observed that neurogenesis occurs in the peri-damaged brain regions after TBI. However, they were unable to confirm whether the newly generated cells originated from local cortical progenitor cells or migrated from neurogenic regions after TBI. According to Richardson et al. (57), assessing the migration of neuroblasts from the subventricular zone to the damaged cortical area on TBI in the human brain becomes difficult due to the long distance between the two regions compared to the rostral migratory route of rodents. Chiaretti et al. (58) showed a significant up-regulation of doublecortin (DCX) level in the cerebrospinal fluid of children with severe TBI that may reflect an attempt at neuroprotection against the biochemical and molecular cascades triggered by traumatic insult.

There are no consistent reports on the effects of PTE in humans with regard to altered neurogenesis. Even more so, there is no consistent evidence indicating what would be the relevance of this altered neurogenesis after TBI in humans to the ensuing PTE.

Mossy fiber sprouting. Mossy fiber sprouting (MFS) was first described in patients with temporal lobe epilepsy and it became an important and frequent histopathological finding replicated in animal models. Mossy fibers normally extend to the hilus with projections to the excitatory and inhibitory interneurons and then run the stratum lucidum to synapse onto pyramidal neurons of the CA3 region. The projection of these axons under physiological conditions normally maintains a balance between inhibition and excitation, but in the epileptic brain, the MFS creates new recurrent excitatory circuits, projecting back to the molecular layer of the dentate gyrus (59). Normally, MFS is first detected within days after seizures or experimental lesions, develops by 2 weeks, and is long lasting. However, there has been controversy about the overall functional significance of sprouted excitatory circuitry as a contributing factor of spontaneous seizure development.

SE models, such as the pilocarpine and KA models, have been used to suggest an association between MFS and epileptogenesis (60). In contrast, Longo et al. (61) demonstrated that in pilocarpine-induced animals the prevention of MFS by cycloheximide (a protein synthesis inhibitor) does not interfere in the development of spontaneous seizures, despite preventing the MFS. In addition, Longo and Mello (62) also demonstrated that cycloheximide can block MFS induced by KA injection, without altering either the frequency or intensity of the behaviorally and/or electroencephalographically recorded ictal and interictal events.

Sutula et al. (63) demonstrated that even brief seizures of kindled rats induces long-lasting structural reorganization in neuronal circuits. MFS develops after a few brief seizures induced by kindling stimulation progressing with repeated seizures (64).

Although MFS is mostly observed in chronic SE models, it may also be seen in TBI models (Figure 1). According to Golarai et al. (65) weight-drop injury leads to a progressive bilateral MFS in the dentate gyrus. Substantial bilateral MFS could also be observed at both acute and chronic time points in FPI model, being more severe ipsilateral to FPI injury, presumably due to the more intense neuronal damage (25). Hunt et al. (66) also observed MFS ipsilateral to $\mathrm{CCl}$ injury at 8-12 weeks after insult. However, while some authors recognize that the MFS is qualitatively less abundant after $\mathrm{TBI}$, compared with the robust sprouting observed after SE (67), according to Hunt et al. (23), MFS after CCI was similar to that observed in humans and other animal PTE models.

With the aim of evaluating the network excitability in the dentate gyrus after TBI, a series of studies using extracellular field recording have been done, but failed to regularly demonstrate epileptiform activity after TBI (23). In addition, Santhakumar et al. (68) showed that rats presented recovery a month after FPI, together with an early increase in granule cell layer extracellular excitability that was related to MFS. Therefore, Hunt et al. (66) suggest that the emergence of MFS may play a different functional role in the dentate gyrus after mechanical TBI compared to pharmacologically-induced PTE models.

In humans, MFS is frequently found in surgical epilepsy tissue (69). The mossy fibers terminals are distributed in the hippocampus of humans with epilepsy 
in a similar way as seen in the kindling model in rats (69). Swartz et al. (70) performed further histologic analyses in tissue from patients with a history of significant head trauma but without any other risk factor for epilepsy, from the California Comprehensive Epilepsy Program database. Ten of 11 hippocampal specimens presented some degree of MFS, and also of granule cell dispersion, both related with hilar neuron loss. Despite the observed reorganization of the mossy fibers, these authors could not correlate the presence of sprouting to the duration of epilepsy, and even less so whether it was a pre-existing condition to the head trauma.

In summary, despite the various studies that investigate MFS, its role in epileptogenesis remains controversial and unclear. MFS is a common finding in epilepsy but not all patients or animal models with spontaneous seizures develop MFS. Taking this into account, MFS has neither a pro-epileptogenic nor an anti-epileptogenic role.

\section{Neuroinflammation}

The induction of cytokines after the occurrence of prolonged and acute seizures has been extensively studied in animal models of seizures and epilepsy. The activation of inflammatory pathways, which leads to the release of mediators, increases the severity of subsequent seizures and epileptogenesis, and supports the hypothesis that inflammation may play a lead role in the pathophysiology of seizures and the associated neuropathology in PTE (71).

Indeed, gliosis, microglia activation, and cytokine production, such as interleukin (IL)-1 $\beta$ and tumor necrosis factor (TNF)- $\alpha$, and consequently neuroinflammation can change neuronal excitability by modulating receptor function and expression, perpetuating the chronic nature of epilepsy (71). Although neuroinflammation is considered to play a key role in the pathophysiology of epilepsy, what are (and whether there are) the critical neuroinflammatory processes underlying epileptogenesis is still unclear. Seizures induced either chemically or electrically could increase cytokines in rodent brain areas involved in the onset of epileptic activity (72). To that end, proinflammatory cytokines are induced in the brain also by kindled seizures (73). Also, Ambrogini et al. (74) demonstrated that KA-induced SE is capable of triggering neuroinflammatory processes in the hippocampus of rats characterized by astrogliosis and microglial activation, in addition to the expression of IL-1 $\beta$ and TNF- $\alpha$.

Comparing the expression of $\mathrm{IL}-1 \beta$ in microglia and astrocyte cells in two different SE models (electrical stimulation and pilocarpine-induced SE), Ravizza et al. (75) observed that during the acute phases of SE, the upregulation of IL-1 $\beta$ was observed in both microglia and astrocytes, whereas only astrocytes showed enhanced immunostaining during epileptogenesis. In the chronic epileptic phase, IL-1 $\beta$ is still observed in astrocytes and also in microglia but only in the electrical stimulation model. These data indicate that the expression of $\mathrm{IL}-1 \beta$ in astrocytes is continuous regardless of seizure frequency, whereas microglia expression depends on ongoing and severe epileptic activity, indicating that brain inflammation is a chronic process developing after the initial precipitating insult that can persist in epileptogenic tissue in the absence of ongoing seizure activity.

Moreover, increased expression of inflammatory genes $(I L-1 \beta, I L-6, T N F-\alpha)$ in the hippocampus and amygdala has been reported in several PTE models such as KA (71) and lithium-pilocarpine (76), representing a generalized molecular response to seizures.

The neuroinflammation induced in TBI models has been reported as similar to that of SE models. According to Dalgard et al. (77), the expression of CXCL-1, IFN- $\gamma$, $\mathrm{TNF}-\alpha, \mathrm{IL}-4$, and IL-13 are increased in ipsilateral cortex at $4 \mathrm{~h}$ after $\mathrm{CCl}$. Increased expression of some cytokines were also reported $24 \mathrm{~h}$ after lateral FPI, both in the ipsilateral and contralateral cortex (78). Interestingly, the same research group reported similar magnitude of expression among the same cytokines $24 \mathrm{~h}$ after lateral FPI and after pilocarpine-induced SE $(78,79)$. Specifically, both models showed the highest expression for CCL2 and similar expression levels for CCL3, CCL5, and TNF- $\alpha$ $(78,79)$. In addition, Su et al. (80) using the FPI model reported that TBI may increase the expression of eotaxin, G-CSF, GM-CSF, GRO/KC, IFN- $\gamma, \mathrm{IL}-1 \alpha$, IL-1 $\beta$, IL-2, IL-4, IL-5, IL-6, IL-10, IL-12, p70, IL-13, IL-17, leptin, MCP-1, TNF- $\alpha$, and VEGF in the area of cortical injury even 7 days after FPI. Residual level of neuroinflammation may also persist chronically $(11,44,78)$.

It is well established that TBI in humans is associated with an inflammatory response that varies according to stimulus intensity, presence of blood in the brain parenchyma, and the presence of penetrating injury, among other aspects (81). However, causal links between inflammatory signaling cascades taking place after TBI and the emergence of seizures and the development of epilepsy have yet to be more firmly established.

In summary, it is well-established that seizures promote an inflammatory immune cascade, although emerging evidence also suggests that neuroinflammation may also be causal to seizures and epilepsy by inducing changes in neuronal function and connectivity, leading to regional hyperexcitability and subsequent seizure susceptibility. Nevertheless there is no distinctive feature that characterizes these processes among PTE models, which in general, display similar cascades of events (Figure 1).

\section{Antiepileptic drugs (AEDs)}

It is known that people who suffer TBI have increased acute seizure susceptibility. In this sense, a series of AEDs are routinely administered in the clinic, soon after $\mathrm{TBI}$, to control acute seizures (occurring within 1-2 weeks from injury). In addition, there are also many clinical trials 
using known AEDs, like carbamazepine, valproic acid, phenobarbital, phenytoin, and other compounds aimed at preventing the development of PTE, but all have failed to prove efficacy (28). Therefore, the use of experimental models to verify the effectiveness of already known AEDs and test new compounds on PTE prevention is of extreme importance.

The kindling model has long been used to study the effects of anticonvulsant drugs on seizure development. Diazepam is more effective at blocking amygdala-kindled seizures compared to seizures induced by cortical stimulation, which are better blocked by procaine hydrochloride and diphenylhydantoin. Phenobarbital is effective in the suppression of hippocampal and amygdala seizures (30). To make the topic even more complex, a single exposure to carbamazepine or lamotrigine $48 \mathrm{~h}$ after stimulation leads to a decrease in the capacity of these AED to mitigate further seizures evoked after discharges (34). According to Löscher (82), in the amygdala-kindling, low doses of lamotrigine during the development phase leads to a reduced anticonvulsant response to the drug in fully kindled rats. Lamotrigine-refractory kindled rats are resistant to topiramate, carbamazepine, and phenytoin, but not felbamate, valproate, and retigabine. During the kindling acquisition phase, some antiepileptogenic drugs (i.e., valproate) given before each stimulus could be sufficient to mitigate or retard the kindling. Song et al. (33) compared the effects of three AEDs in mice submitted to kindling models. Levetiracetam and phenytoin abolished spontaneous recurrent seizures in 6 of 6 and 5 of 6 mice, respectively. Lorazepam, in turn, was effective in decreasing the motor seizures severity in all mice, but it was not enough to reduce the duration and incidence of associated afterdischarges in the hippocampus.

Also, many compounds have been tested in TBI models. According to Pitkanen and Mclntosh (28), AEDs used in clinics, such as carbamazepine, phenobarbital, valproate, phenytoin, clonazepam, zonisamide, primidone, and ethosuximide, can all suppress spontaneous seizures induced by ferric chloride, but again, with no translational benefit proven.

In the FPI model, rats acutely treated with tacrolimus showed a reduction in the number of non-convulsive seizures compared to untreated rats, even presenting a similar degree of cortical atrophy (6). In addition, slices from rats treated with felbamate after FPI exhibited long-term potentiation in CA1, which was suppressed in untreated animals. This fact suggests a possible neuroprotective property of felbamate against TBI (25). The administration of topiramate 30 min after FPI proved beneficial to sensorimotor behavior, even though it was not favorable on learning or neuronal survival. Other drugs used after FPI such as lacosamide had no effect on lesion size, anatomical damage, motor impairment, or functional recovery (83). According to Dash et al. (84), the administration of valproate starting at $30 \mathrm{~min}$ or $3 \mathrm{~h}$ after $\mathrm{CCl}$ promoted improvement of blood-brain barrier (BBB) integrity, decrease of hippocampal dendritic damage, improved spatial memory and motor function, and lessened cortical contusion volume. High doses of ethosuximide and phenytoin administered $30 \mathrm{~min}$ after penetrating ballistic-like brain injury attenuated spontaneous nonconvulsive seizures, but lower doses failed to have the same effect (85). The clinical relevance of such findings, however, is hampered by the extremely short time interval between injury and drug administration.

Leite and Cavalheiro (86) tested the effect of five AEDs in the pilocarpine model. Phenobarbital, phenytoin, and carbamazepine were effective against spontaneous seizures. Valproic acid was also effective against spontaneous seizures, but only at the high doses. Last, but not the least, ethosuximide was not effective against spontaneous seizures. In accordance, Glien et al. (87) also showed that levetiracetam decreases spontaneous recurrent seizures frequency in the rat pilocarpine model.

In the KA model, valproate, carbamazepine, and lamotrigine were effective to dose-dependently suppress hippocampal paroxysmal discharges (HPDs) and modified EEG activity and behavior. At high doses, levetiracetam and pregabalin were effective to suppress HPDs, but did not change any behavior or EEG activity. Furthermore, dose-dependent diazepam, tiagabine, phenobarbital, and vigabatrin suppressed HPDs, but did no other change (88).

It is now clear that the so-called AEDs are in fact drugs that affect the expression of seizures but do not affect the development of epileptogenesis. Despite their name, AEDs do not prevent epilepsy but only its expression (e.g., seizures). Indeed, as discussed in the previous sections of this review, the lesion that may trigger PTE, regardless of its nature, sets in motion a similar cascade of events that overlaps with other models of epilepsy. Thus, drug treatment tested on these other epilepsy models with a higher frequency of recurrent spontaneous seizures would ultimately have great predictive value for PTE (even when not using isomorphic models to TBI).

To date, neither TBI or SE models (but see below) have provided evidence of compounds that would effectively alter epileptogenesis after TBI. The predictive capability that could be expected from models with a stronger etiological basis has not yet been demonstrated. The fact that the emergence of spontaneous seizures is a rare event in TBI models does not help in providing a platform for testing of such compounds.

Research from our group has provided evidence that anticholinergic agents might provide an effective means to modify disease progression in the pilocarpine model of epilepsy $(89,90)$. The administration of scopolamine starting $3 \mathrm{~h}$ after the onset of pilocarpine-induced SE was effective in altering epileptogenesis (90). Similarly, in a more extensive study, the administration of biperiden (another cholinergic antagonist) was also shown to be effective in altering disease progression in the same 
model (89). We concluded that anti-muscarinic agents or, more broadly, drugs that interfere with plastic processes may be potentially relevant in the quest for diseasemodifying agents that may diminish the burden of PTE.

\section{Final remarks}

We put forward the suggestion that the ideal model to investigate potential therapies against PTE has yet to be defined. In fact, for numerous aspects, TBI models mimicking the mechanical traumatic injury such as $\mathrm{CCl}$, FPI, blast, and so on are the most faithful to recapitulate the sequence of events after TBI. However, the diminished capacity of those models to easily promote PTE also hampers their ability to provide more useful instruments in the screening of new compounds that may influence epileptogenesis. Here, we addressed the main differences and similarities between typical TBI models, kindling, and SE models with regard to the emergence of spontaneous seizures, seizure response to drugs, neuroinflammation, and other features often studied in animal models and human epilepsy, most notably PTE. Currently,

\section{References}

1. Hyder AA, Wunderlich CA, Puvanachandra P, Gururaj G, Kobusingye OC. The impact of traumatic brain injuries: a global perspective. NeuroRehabilitation 2007; 22: 341-353, doi: 10.3233/NRE-2007-22502.

2. GBD 2016 Traumatic Brain Injury and Spinal Cord Injury Collaborators. Global, regional, and national burden of traumatic brain injury and spinal cord injury, 1990-2016: a systematic analysis for the Global Burden of Disease Study 2016. Lancet Neurol 2019; 18: 56-87, doi: 10.1016/S14744422(18)30415-0.

3. Roozenbeek B, Maas Al, Menon DK. Changing patterns in the epidemiology of traumatic brain injury. Nat Rev Neurol 2013; 9: 231-236, doi: 10.1038/nrneurol.2013.22.

4. Xiong $\mathrm{Y}$, Mahmood A, Chopp M. Animal models of traumatic brain injury. Nat Rev Neurosci 2013; 14: 128-142, doi: 10. 1038/nrn3407.

5. Capizzi A, Woo J, Verduzco-Gutierrez M. Traumatic brain injury: an overview of epidemiology, pathophysiology, and medical management. Med Clin North Am 2020; 104: 213238, doi: 10.1016/j.mcna.2019.11.001.

6. Campbell JN, Gandhi A, Singh B, Churn SB. Traumatic brain injury causes a tacrolimus-sensitive increase in nonconvulsive seizures in a rat model of post-traumatic epilepsy. Int J Neurol Brain Disord 2014; 1: 1-11, doi: 10.15436/ 2377-1348.14.002.

7. Fisher RS, Acevedo C, Arzimanoglou A, Bogacz A, Cross $\mathrm{JH}$, Elger CE, et al. ILAE official report: a practical clinical definition of epilepsy. Epilepsia 2014; 55: 475-482, doi: 10.1111/epi.12550.

8. Hauser WA, Annegers JF, Kurland LT. Prevalence of epilepsy in Rochester, Minnesota: 1940-1980. Epilepsia 1991; 32: 429-445, doi: 10.1111/j.1528-1157.1991.tb04675.x. the main practical application of any model of PTE is that of providing a means to prevent or modify disease progression after TBI. Here, we provide evidence that, except for the causative (etiological) aspect of TBI models, there is no distinctive pathological feature that is exclusively present in those models and not in the kindling or SE models. We put forward the hypothesis that the use of various modeling strategies, and not only those restricted to mechanical traumatic injury, might allow for a broader understanding of the different mechanisms of seizure induction, propagation, and occurrence and could help the search for biomarkers and new drugs to prevent PTE.

\section{Acknowledgments}

This study was supported by Fundação de Amparo à Pesquisa do Estado de São Paulo (FAPESP; grant 2018/ 24561-5), Coordenação de Aperfeiçoamento de Pessoal de Nível Superior, Brazil (CAPES; Finance Code 001), and Conselho Nacional de Desenvolvimento Científico e Tecnológico (CNPq; grant 311619/2019-3).

9. Raymont V, Salazar AM, Lipsky R, Goldman D, Tasick G, Grafman J. Correlates of posttraumatic epilepsy 35 years following combat brain injury. Neurology 2010; 75: 224-229, doi: 10.1212/WNL.0b013e3181e8e6d0.

10. Haltiner AM, Temkin NR, Dikmen SS. Risk of seizure recurrence after the first late posttraumatic seizure. Arch Phys Med Rehabil 1997; 78: 835-840, doi: 10.1016/S00039993(97)90196-9.

11. Morganti-Kossmann MC, Satgunaseelan L, Bye N, Kossmann T. Modulation of immune response by head injury. Injury 2007; 38: 1392-1400, doi: 10.1016/j.injury.2007.10.005.

12. Pitkänen A, Lukasiuk K. Mechanisms of epileptogenesis and potential treatment targets. Lancet Neurol 2011; 10: 173186, doi: 10.1016/S1474-4422(10)70310-0.

13. Curia G, Longo D, Biagini G, Jones RS, Avoli M. The pilocarpine model of temporal lobe epilepsy. J Neurosci Methods 2008; 172: 143-157, doi: 10.1016/j.jneumeth.2008.04.019.

14. Willmore LJ, Sypert GW, Munson JB. Recurrent seizures induced by cortical iron injection: a model of posttraumatic epilepsy. Ann Neurol 1978; 4: 329-336, doi: 10.1002/ ana.410040408.

15. Engstrom ER, Hillered L, Flink R, Kihlstrom L, Lindquist C, Nie JX, et al. Extracellular amino acid levels measured with intracerebral microdialysis in the model of posttraumatic epilepsy induced by intracortical iron injection. Epilepsy Res 2001; 43: 135-144, doi: 10.1016/S0920-1211(00)00191-1.

16. Hazra R, Ray K, Guha D. Inhibitory role of Acorus calamus in ferric chloride-induced epileptogenesis in rat. Hum Exp Toxicol 2007; 26: 947-953, doi: 10.1177/096032710708 7791.

17. Williams AJ, Ling GS, Tortella FC. Severity level and injury track determine outcome following a penetrating ballistic-like 
brain injury in the rat. Neurosci Lett 2006; 408: 183-188, doi: 10.1016/j.neulet.2006.08.086.

18. Keith $\mathrm{KA}$, Huang $\mathrm{JH}$. Animal models of post-traumatic epilepsy. Diagnostics (Basel) 2019; 10: 4, doi: 10.3390/diagnostics 10010004.

19. D'Ambrosio R, Perucca E. Epilepsy after head injury. Curr Opin Neurol 2004; 17: 731-735, doi: 10.1097/00019052200412000-00014.

20. Kharatishvili I, Nissinen JP, Mclntosh TK, Pitkanen A A model of posttraumatic epilepsy induced by lateral fluidpercussion brain injury in rats. Neuroscience 2006; 140: 685-697, doi: 10.1016/j.neuroscience.2006.03.012.

21. Lighthall JW. Controlled cortical impact: a new experimental brain injury model. J Neurotrauma 1988; 5: 1-15, doi: 10.1089/neu.1988.5.1.

22. Kelly KM, Miller ER, Lepsveridze E, Kharlamov EA, McHedlishvili Z. Posttraumatic seizures and epilepsy in adult rats after controlled cortical impact. Epilepsy Res 2015; 117: 104-116, doi: 10.1016/j.eplepsyres.2015.09.009.

23. Hunt RF, Scheff SW, Smith BN. Posttraumatic epilepsy after controlled cortical impact injury in mice. Exp Neurol 2009; 215: 243-252, doi: 10.1016/j.expneurol.2008.10.005.

24. Song $H$, Cui J, Simonyi A, Johnson CE, Hubler GK, DePalma RG, et al. Linking blast physics to biological outcomes in mild traumatic brain injury: Narrative review and preliminary report of an open-field blast model. Behav Brain Res 2018, 340: 147-158, doi: 10.1016/j.bbr.2016. 08.037.

25. Glushakov AV, Glushakova OY, Dore S, Carney PR, Hayes RL. Animal models of posttraumatic seizures and epilepsy. Methods Mol Biol 2016, 1462: 481-519, doi: 10.1007/978-14939-3816-2.

26. Lu XC, Mountney A, Chen Z, Wei G, Cao Y, Leung LY, et al. Similarities and differences of acute nonconvulsive seizures and other epileptic activities following penetrating and ischemic brain injuries in rats. J Neurotrauma 2013; 30: 580-590, doi: 10.1089/neu.2012.2641.

27. Bugay V, Bozdemir E, Vigil FA, Chun SH, Holstein DM, Elliott WR, et al. A mouse model of repetitive blast traumatic brain injury reveals post-trauma seizures and increased neuronal excitability. J Neurotrauma 2020; 37: 248-261, doi: 10.1089/neu.2018.6333.

28. Pitkanen A, Mclntosh TK. Animal models of post-traumatic epilepsy. J Neurotrauma 2006; 23: 241-261, doi: 10.1089/ neu.2006.23.241.

29. Goddard GV. Development of epileptic seizures through brain stimulation at low intensity. Nature 1967; 214: 10201021, doi: 10.1038/2141020a0.

30. Coppola A, Moshe SL. Animal models. Handb Clin Neurol 2012; 107: 63-98, doi: 10.1016/B978-0-444-52898-8.00004-5.

31. Brandt C, Ebert U, Loscher W. Epilepsy induced by extended amygdala-kindling in rats: lack of clear association between development of spontaneous seizures and neuronal damage. Epilepsy Res 2004; 62: 135-156, doi: 10.1016/ j.eplepsyres.2004.08.008.

32. Pinel JP, Cheung KF. Controlled demonstration of metrazol kindling. Pharmacol Biochem Behav 1977; 6: 599-600, doi: 10.1016/0091-3057(77)90124-1.

33. Song H, Tufa U, Chow J, Sivanenthiran N, Cheng C, Lim S, et al. Effects of antiepileptic drugs on spontaneous recurrent seizures in a novel model of extended hippocampal kind- ling in mice. Front Pharmacol 2018; 9: 451, doi: 10.3389/ fphar.2018.00451.

34. Kandratavicius L, Balista PA, Lopes-Aguiar C, Ruggiero RN, Umeoka EH, Garcia-Cairasco N, et al. Animal models of epilepsy: use and limitations. Neuropsychiatr Dis Treat 2014, 10: 1693-1705, doi: 10.2147/NDT.S50371.

35. Lothman EW, Hatlelid JM, Zorumski CF, Conry JA, Moon PF, Perlin JB. Kindling with rapidly recurring hippocampal seizures. Brain Res 1985, 360: 83-91, doi: 10.1016/00068993(85)91223-5.

36. McIntyre DC, Nathanson D, Edson N. A new model of partial status epilepticus based on kindling. Brain Res 1982; 250: 53-63, doi: 10.1016/0006-8993(82)90952-0.

37. Cela E, McFarlan AR, Chung AJ, Wang T, Chierzi S, Murai KK, et al. An optogenetic kindling model of neocortical epilepsy. Sci Rep 2019; 9: 5236, doi: 10.1038/s41598-019-41533-2.

38. Ben-Ari Y, Tremblay E, Riche D, Ghilini G, Naquet R. Electrographic, clinical and pathological alterations following systemic administration of kainic acid, bicuculline or pentetrazole: metabolic mapping using the deoxyglucose method with special reference to the pathology of epilepsy. Neuroscience 1981; 6: 1361-1391, doi: 10.1016/0306-4522(81)90193-7.

39. Nissinen J, Halonen T, Koivisto E, Pitkänen A. A new model of chronic temporal lobe epilepsy induced by electrical stimulation of the amygdala in rat. Epilepsy Res 2000; 38: 177-205, doi: 10.1016/S0920-1211(99)00088-1.

40. Turski WA, Cavalheiro EA, Schwarz M, Czuczwar SJ, Kleinrok Z, Turski L. Limbic seizures produced by pilocarpine in rats: behavioural, electroencephalographic and neuropathological study. Behav Brain Res 1983; 9: 315-335, doi: 10.1016/0166-4328(83)90136-5.

41. Scorza FA, Arida RM, Naffah-Mazzacoratti MG, Scerni DA, Calderazzo L, Cavalheiro EA. The pilocarpine model of epilepsy: what have we learned? An Acad Bras Cienc 2009; 81: 345-365, doi: 10.1590/S0001-37652009000300003.

42. Covolan L, Mello LE. Temporal profile of neuronal injury following pilocarpine or kainic acid-induced status epilepticus. Epilepsy Res 2000; 39: 133-152, doi: 10.1016/S09201211(99)00119-9.

43. Washington PM, Forcelli PA, Wilkins T, Zapple DN, Parsadanian M, Burns MP. The effect of injury severity on behavior: a phenotypic study of cognitive and emotional deficits after mild, moderate, and severe controlled cortical impact injury in mice. J Neurotrauma 2012; 29: 2283-2296, doi: 10.1089/neu.2012.2456.

44. Loane DJ, Kumar A, Stoica BA, Cabatbat R, Faden Al. Progressive neurodegeneration after experimental brain trauma: association with chronic microglial activation. $J$ Neuropathol Exp Neurol 2014; 73: 14-29, doi: 10.1097/NEN.000000000 0000021

45. Parent JM, Yu TW, Leibowitz RT, Geschwind DH, Sloviter $\mathrm{RS}$, Lowenstein $\mathrm{DH}$. Dentate granule cell neurogenesis is increased by seizures and contributes to aberrant network reorganization in the adult rat hippocampus. $J$ Neurosci 1997; 17: 3727-3738, doi: 10.1523/JNEUROSCI.17-1003727.1997.

46. Mello LE, Cavalheiro EA, Tan AM, Kupfer WR, Pretorius JK, Babb TL, et al. Circuit mechanisms of seizures in the pilocarpine model of chronic epilepsy: cell loss and mossy fiber sprouting. Epilepsia 1993; 34: 985-995, doi: 10.1111/ j.1528-1157.1993.tb02123.x. 
47. Parent JM, Janumpalli S, McNamara JO, Lowenstein DH. Increased dentate granule cell neurogenesis following amygdala kindling in the adult rat. Neurosci Lett 1998; 247: 9-12, doi: 10.1016/S0304-3940(98)00269-9.

48. Jessberger S, Parent JM. Epilepsy and adult neurogenesis. Cold Spring Harb Perspect Biol 2015; 7: a020677, doi: 10.1101/cshperspect.a020677.

49. Zellinger C, Seeger N, Hadamitzky M, Fischborn S, Russmann $\mathrm{V}$, Wendt $\mathrm{H}$, et al. Impact of the erythropoietinderived peptide mimetic Epotris on the histopathological consequences of status epilepticus. Epilepsy Res 2011; 96: 241-249, doi: 10.1016/j.eplepsyres.2011.06.009.

50. Danzer SC. Depression, stress, epilepsy and adult neurogenesis. Exp Neurol 2012; 233: 22-32, doi: 10.1016/j. expneurol.2011.05.023.

51. Bonde S, Ekdahl CT, Lindvall O. Long-term neuronal replacement in adult rat hippocampus after status epilepticus despite chronic inflammation. Eur J Neurosci 2006; 23: 965-974, doi: 10.1111/j.1460-9568.2006.04635.x.

52. Neuberger EJ, Swietek B, Corrubia L, Prasanna A, Santhakumar V. Enhanced dentate neurogenesis after brain injury undermines long-term neurogenic potential and promotes seizure susceptibility. Stem Cell Reports 2017; 9: 972-984, doi: 10.1016/j.stemcr.2017.07.015.

53. Hattiangady B, Rao MS, Shetty AK. Chronic temporal lobe epilepsy is associated with severely declined dentate neurogenesis in the adult hippocampus. Neurobiol Dis 2004; 17: 473-490, doi: 10.1016/j.nbd.2004.08.008.

54. Scharfman HE, Gray WP. Relevance of seizure-induced neurogenesis in animal models of epilepsy to the etiology of temporal lobe epilepsy. Epilepsia 2007, 48: 33-41, doi: 10.1111/j.1528-1167.2007.01065.x.

55. Mohapel P, Ekdahl CT, Lindvall O. Status epilepticus severity influences the long-term outcome of neurogenesis in the adult dentate gyrus. Neurobiol Dis 2004; 15: 196-205, doi: 10.1016/j.nbd.2003.11.010.

56. Zheng W, ZhuGe Q, Zhong M, Chen G, Shao B, Wang H, et al. Neurogenesis in adult human brain after traumatic brain injury. J Neurotrauma 2013; 30: 1872-1880, doi: 10. 1089/neu.2010.1579.

57. Richardson RM, Sun D, Bullock MR. Neurogenesis after traumatic brain injury. Neurosurg Clin N Am 2007; 18: 169181, xi., doi: 10.1016/j.nec.2006.10.007.

58. Chiaretti A, Antonelli A, Genovese O, Pezzotti P, Rocco CD, Viola L, et al. Nerve growth factor and doublecortin expression correlates with improved outcome in children with severe traumatic brain injury. J Trauma 2008; 65: 8085, doi: 10.1097/TA.0b013e31805f7036.

59. Dudek FE, Staley KJ. Laser scanning photostimulation: new evidence for enhanced recurrent excitation in a model of posttraumatic epilepsy. Epilepsy Curr 2006; 6: 215-216, doi: 10.1111/j.1535-7511.2006.00150.x.

60. Lemos T, Cavalheiro EA. Suppression of pilocarpineinduced status epilepticus and the late development of epilepsy in rats. Exp Brain Res 1995, 102: 423-428, doi: 10.1007/BF00230647.

61. Longo BM, Mello LE. Blockade of pilocarpine- or kainateinduced mossy fiber sprouting by cycloheximide does not prevent subsequent epileptogenesis in rats. Neurosci Lett 1997; 226: 163-166, doi: 10.1016/S0304-3940(97)00 267-X
62. Longo BM, Mello LE. Supragranular mossy fiber sprouting is not necessary for spontaneous seizures in the intrahippocampal kainate model of epilepsy in the rat. Epilepsy Res 1998, 32: 172-182, doi: 10.1016/S0920-1211(98)00049-7.

63. Sutula TP, Kotloski RJ. Kindling: a model and phenomenon of epilepsy. In: Pitkänen A, Buckmaster PS, Galanopoulou AS, Moshé LS (Editors). Models of seizures and epilepsy. 2nd edn. London: Academic Press; 2017: 813-825, doi: 10. 1016/B978-0-12-804066-9.00055-9.

64. Nissinen J, Lukasiuk K, Pitkanen A. Is mossy fiber sprouting present at the time of the first spontaneous seizures in rat experimental temporal lobe epilepsy? Hippocampus 2001; 11: 299-310, doi: 10.1002/hipo.1044.

65. Golarai G, Greenwood AC, Feeney DM, Connor JA. Physiological and structural evidence for hippocampal involvement in persistent seizure susceptibility after traumatic brain injury. J Neurosci 2001; 21: 8523-8537, doi: 10.1523/JNEUROSCI.21-21-08523.2001.

66. Hunt RF, Scheff SW, Smith BN. Regionally localized recurrent excitation in the dentate gyrus of a cortical contusion model of posttraumatic epilepsy. J Neurophysiol 2010, 103: 1490-1500, doi: 10.1152/jn.00957.2009.

67. Traub RD, Wong RK. Cellular mechanism of neuronal synchronization in epilepsy. Science 1982; 216: 745-747, doi: 10.1126/science.7079735.

68. Santhakumar V, Bender R, Frotscher M, Ross ST, Hollrigel GS, Toth Z, et al. Granule cell hyperexcitability in the early post-traumatic rat dentate gyrus: the 'irritable mossy cell' hypothesis. J Physiol 2000; 524 Pt 1: 117-134, doi: 10.1111/ j.1469-7793.2000.00117.x.

69. Sutula T, Cascino G, Cavazos J, Parada I, Ramirez L. Mossy fiber synaptic reorganization in the epileptic human temporal lobe. Ann Neurol 1989; 26: 321-330, doi: 10.1002/ ana.410260303.

70. Swartz BE, Houser CR, Tomiyasu U, Walsh GO, DeSalles A, Rich JR, et al. Hippocampal cell loss in posttraumatic human epilepsy. Epilepsia 2006, 47: 1373-1382, doi: 10.1111/j.1528-1167.2006.00602.x.

71. Vezzani A, Granata T. Brain inflammation in epilepsy: experimental and clinical evidence. Epilepsia 2005; 46: 1724-1743, doi: 10.1111/j.1528-1167.2005.00298.x.

72. Jankowsky JL, Patterson PH. The role of cytokines and growth factors in seizures and their sequelae. Prog Neurobiol 2001; 63: 125-149, doi: 10.1016/S0301-0082(00) 00022-8.

73. Plata-Salaman CR, Ilyin SE, Turrin NP, Gayle D, Flynn MC, Romanovitch $\mathrm{AE}$, et al. Kindling modulates the IL-1beta system, TNF-alpha, TGF-beta1, and neuropeptide mRNAs in specific brain regions. Brain Res Mol Brain Res 2000; 75: 248-258, doi: 10.1016/S0169-328X(99)00306-X.

74. Ambrogini P, Albertini MC, Betti M, Galati C, Lattanzi D, Savelli D, et al. Neurobiological correlates of alphatocopherol antiepileptogenic effects and microRNA expression modulation in a rat model of kainate-induced seizures. Mol Neurobiol 2018; 55: 7822-7838, doi: 10.1007/s12035018-0946-7.

75. Ravizza T, Gagliardi B, Noe F, Boer K, Aronica E, Vezzani A. Innate and adaptive immunity during epileptogenesis and spontaneous seizures: evidence from experimental models and human temporal lobe epilepsy. Neurobiol Dis 2008, 29: 142-160, doi: 10.1016/j.nbd.2007.08.012. 
76. Voutsinos-Porche B, Koning E, Kaplan H, Ferrandon A, Guenounou M, Nehlig A, et al. Temporal patterns of the cerebral inflammatory response in the rat lithium-pilocarpine model of temporal lobe epilepsy. Neurobiol Dis 2004; 17: 385-402, doi: 10.1016/j.nbd.2004.07.023.

77. Dalgard CL, Cole JT, Kean WS, Lucky JJ, Sukumar G, McMullen DC, et al. The cytokine temporal profile in rat cortex after controlled cortical impact. Front Mol Neurosci 2012; 5: 6, doi: 10.3389/fnmol.2012.00006.

78. Mukherjee S, Katki K, Arisi GM, Foresti ML, Shapiro LA. Early TBI-induced cytokine alterations are similarly detected by two distinct methods of multiplex assay. Front $\mathrm{Mol}$ Neurosci 2011; 4: 21, doi: 10.3389/fnmol.2011.00021.

79. Arisi GM, Foresti ML, Katki K, Shapiro LA. Increased CCL2, CCL3, CCL5, and IL-1beta cytokine concentration in piriform cortex, hippocampus, and neocortex after pilocarpineinduced seizures. J Neuroinflammation 2015; 12: 129, doi: 10.1186/s12974-015-0347-z.

80. Su Y, Fan W, Ma Z, Wen X, Wang W, Wu Q, et al. Taurine improves functional and histological outcomes and reduces inflammation in traumatic brain injury. Neuroscience 2014; 266: 56-65, doi: 10.1016/j.neuroscience.2014.02.006.

81. Shi K, Zhang J, Dong JF, Shi FD. Dissemination of brain inflammation in traumatic brain injury. Cell Mol Immunol 2019; 16: 523-530, doi: 10.1038/s41423-019-0213-5.

82. Löscher W. Critical review of current animal models of seizures and epilepsy used in the discovery and development of new antiepileptic drugs. Seizure 2011; 20: 359-368, doi: 10.1016/j.seizure.2011.01.003.

83. Pitkanen A, Lukasiuk K. Molecular and cellular basis of epileptogenesis in symptomatic epilepsy. Epilepsy Behav 2009; 14: 16-25, doi: 10.1016/j.yebeh.2008.09.023.
84. Dash PK, Orsi SA, Zhang M, Grill RJ, Pati S, Zhao J, et al. Valproate administered after traumatic brain injury provides neuroprotection and improves cognitive function in rats. PLOS One 2010; 5: e11383, doi: 10.1371/journal.pone.0011383.

85. Mountney A, Shear DA, Potter B, Marcsisin SR, Sousa J, Melendez $\mathrm{V}$, et al. Ethosuximide and phenytoin dosedependently attenuate acute nonconvulsive seizures after traumatic brain injury in rats. J Neurotrauma 2013; 30: 1973-1982, doi: 10.1089/neu.2013.3001.

86. Leite JP, Cavalheiro EA. Effects of conventional antiepileptic drugs in a model of spontaneous recurrent seizures in rats. Epilepsy Res 1995; 20: 93-104, doi: 10.1016/0920-1211(94) 00070-D.

87. Glien M, Brandt C, Potschka H, Loscher W. Effects of the novel antiepileptic drug levetiracetam on spontaneous recurrent seizures in the rat pilocarpine model of temporal lobe epilepsy. Epilepsia 2002, 43: 350-357, doi: 10.1046/ j.1528-1157.2002.18101.x.

88. Duveau V, Pouyatos B, Bressand K, Bouyssieres C, Chabrol $T$, Roche $Y$, et al. Differential effects of antiepileptic drugs on focal seizures in the intrahippocampal kainate mouse model of mesial temporal lobe epilepsy. CNS Neurosci Ther 2016; 22: 497-506, doi: 10.1111/cns.12523.

89. Bittencourt S, Ferrazoli E, Valente MF, Romariz S, Janisset $\mathrm{N}$, Macedo CE, et al. Modification of the natural progression of epileptogenesis by means of biperiden in the pilocarpine model of epilepsy. Epilepsy Res 2017; 138: 88-97, doi: 10.1016/j.eplepsyres.2017.10.019.

90. Pereira HA, Benassi SK, Mello LE. Plastic changes and disease-modifying effects of scopolamine in the pilocarpine model of epilepsy in rats. Epilepsia 2005; 46: 118-124, doi: 10.1111/j.1528-1167.2005.01017.x. 